\title{
Role of Low-dose Human Chorionic Gonadotropin in Follicular Phase for Thin Endometrium in Frozen Embryo Replacement Cycles in in vitro Fertilization/ Intracytoplasmic Sperm Injection Patients: A Pilot Study
}

\author{
${ }^{1}$ Shipra Nigam, ${ }^{2}$ Kundavi Shankar, ${ }^{3}$ Thankam R Varma
}

\begin{abstract}
Introduction: One of the most challenging problems in in vitro fertilization (IVF) is patient with thin endometrium. The objective of the study was to ascertain whether daily human chorionic gonadotropin (hCG) for 7 days with estrogen in hormone replacement frozen embryo transfer (FET) cycles during follicular phase can increase the endometrial thickness (ET) and reduce the cancellation of cycles.
\end{abstract}

Materials and methods: Twenty-five infertile patients with resistant thin endometrium who had antagonist protocol and planned for frozen embryo replacement were recruited. These patients had prior attempts to thicken their endometrium which had failed. All the patients received estrogen daily from D2/3 of cycle. On day 8 or 9 of estrogen administration, $200 \mathrm{IU}$ of hCG was given daily for 7 days. After 7 days on hCG priming (D14/15), ET was measured and progesterone was started accordingly. Identification of an intrauterine gestational sac with fetal heart beat by transvaginal ultrasonography constituted clinical pregnancy.

Results: Mean ET increased significantly from 5.84 to $7.61 \mathrm{~mm}$ ( $p<0.01$ ). About $72 \%$ of patients had more than $20 \%$ improvement in their ET after hCG priming. About $76 \%$ achieved an ET more than $7 \mathrm{~mm}$. Overall, $50 \%$ became pregnant. The ongoing pregnancy rate was $40 \%$.

Conclusion: A total of 200 IU hCG endometrial priming for 7 days in the proliferative phase of hormone replacement cycles for FET is a highly promising approach to thicken thin endometrium with failed prior attempts.

Keywords: Human chorionic gonadotropin in in vitro fertilization/ intracytoplasmic sperm injection cycles, Human chorionic gonadotropin, Thin endometrium.

How to cite this article: Nigam S, Shankar K, Varma TR. Role of Low-dose Human Chorionic Gonadotropin in Follicular Phase for Thin Endometrium in Frozen Embryo Replacement Cycles in in vitro Fertilization/Intracytoplasmic Sperm Injection Patients: A Pilot Study. Int J Infertil Fetal Med 2017;8(3):101-105.

\footnotetext{
${ }^{1}$ Fellow, ${ }^{2}$ Consultant, ${ }^{3}$ Medical Director

${ }^{1-3}$ Department of Reproductive Medicine and Women's Health The Madras Medical Mission, Chennai, Tamil Nadu, India

Corresponding Author: Shipra Nigam, Fellow, Department of Reproductive Medicine and Women's Health, The Madras Medical Mission, Chennai, Tamil Nadu, India, Phone: +914466738000 e-mail: docshipranigam@rediffmail.com
}

Source of support: Nil

Conflict of interest: None

Date of received: 21/06/2017

Date of acceptance: 17/07/2017

Date of publication: November 2017

\section{INTRODUCTION}

The endometrium is essential for implantation and as such the thickness of the endometrium has been always considered as an index of quality, especially in assisted reproduction where the selected embryos should be transferred ideally in a receptive environment. ${ }^{1}$ A thin endometrium is encountered infrequently $(2.4 \%)$ in assisted reproductive technology cycles. When it does occur, it is a cause for concern as it is associated with lower implantation rate and pregnancy rate. Though pregnancies have been reported at 4 and $5 \mathrm{~mm}$ of endometrium, it is apparent that an ET $<6 \mathrm{~mm}$ is associated with a trend toward lower probability of pregnancy. However, no agreement has been reached on ET, although most clinicians empirically prefer endometrium $>7 \mathrm{~mm}$.

Regarding the etiology, it is considered mainly idiopathic; however, a postcurettage surgical complication might be identified in some cases. ${ }^{2}$

Numerous treatments have been tried to improve refractory endometrium, but success has been limited. Current evidence-based medicine has not validated any specific treatment. Regarding improvement of ET and receptivity, in the luteal phase, ever since the early IVF days, drug supplements have been employed in order to increase endometrial receptivity and, thus, to enhance pregnancy achievement. ${ }^{3}$ Regarding the proliferative phase, several ways of treatment have been undertaken to circumvent thin endometrium in order to increase ET, but they showed discordant results.

It has been demonstrated that granulocyte colonystimulating factor (G-CSF) can increase the mesenchymal and hematopoietic stem cells in the bone marrow. ${ }^{4}$ The rationale for intrauterine G-CSF instillation is to improve endometrial growth stems from the understanding that 
the human endometrium contains a small population of mesenchymal stem-like cells that could be responsible for endometrial cyclical growth and reconstruction. Gleicher et $\mathrm{al}^{5}$ were the first to report that intrauterine G-CSF instillation improves ET. Subsequently, many studies on intrauterine G-CSF instillation were published with some reporting improvement, ${ }^{6,7}$ while others showed no difference. 8,9 The only study that reported a significant increase in implantation rate and pregnancy rate performed FET and had younger patients in their study. ${ }^{10}$ Drugs that increase endometrial blood flow have been administered individually or in combination to improve ET. None of these therapies, such as pentoxifylline $800 \mathrm{mg} /$ day and tocopherol 1,000 mg/day given over several months, ${ }^{11}$ sildenafil $100 \mathrm{mg} /$ day given as vaginal pessary, L-arginine $6 \mathrm{gm} / \mathrm{day}^{12}$ and low-dose aspirin $75 \mathrm{mg}$ /day have met with much success.

In this pilot study, subjects with repeatedly resistant thin endometrium $<7 \mathrm{~mm}$ were recruited. We sought to investigate the possible role of adding low-dose hCG in the follicular phase, on the endometrial growth and development.

\section{MATERIALS AND METHODS}

The study was conducted at the Institute of Reproductive Medicine and Women's Health, The Madras Medical Mission, Chennai, Tamil Nadu, India, after obtaining approval from Institute's Ethical Committee. Twentyfive patients who were planned for fresh donor or frozen embryos were recruited. Informed consent was taken from all the patients.

The inclusion criteria were

- ET below $7 \mathrm{~mm}$, consistently in previous IVF treatments including frozen embryo

- Failure of previous medications (oral estrogens, vaginal sildenafil, vitamin E)

- Fresh donor or frozen embryo replacement cycles

- Hysteroscopy performed with intact endometrial cavity.

All patients were started on estrogen on day $2 / 3$ of their periods. All of them had blood estradiol $<50 \mathrm{pg} / \mathrm{mL}$. Estradiol valerate $2 \mathrm{mg}$ thrice daily was given for 5 days followed by $8 \mathrm{mg}$ per day, thereafter. On day 8 or 9 of the cycle along with continuing $8 \mathrm{mg}$ estrogen per day, injections of hCG (200 IU) were given daily for 7 days. After a week on hCG priming (day 14 or 15 of the cycle), ET was measured with transvaginal ultrasound. The hCG priming was discontinued and from the next day progesterone administration was initiated if ET was $>7 \mathrm{~mm}$. The luteal phase support consisted of $8 \mathrm{mg}$ of estradiol valerate per day (in four divided doses) along with $800 \mathrm{mg}$ micronized progesterone per vaginally (in two doses) and oral progesterone (Duphaston $10 \mathrm{mg}$ ) thrice daily.
All ultrasounds were performed by the same operator. All embryo transfers were performed by the same operator. Measurement of the endometrium was performed under high magnification, on the longitudinal plane of the uterus and at the thicker area. Since objectivity of ultrasonic measurement of ET might still be questionable due to intraobserver bias, we calculated more than $10 \%$ increase (still subjective) and $>20 \%$ improvement (more objective) in ET when comparing the results.

Pregnancy test was performed 16 days after progesterone initiation. Clinical pregnancy was considered as presence of gestational sac with fetal heart beat at 7 weeks. Continuous variables were compared using paired sample t-test. Categorical variables were compared using Fisher's exact test. The significance level was set at 5\% (p<0.05).

\section{RESULTS}

A total of 25 patients were recruited for the present study. The mean age was 38 years. Mean ET in the previous estradiol primed cycle on D8 and D14/15 was $5.49 \mathrm{~mm}$ (4.1-6.7 $\mathrm{mm}$ ) and $5.92 \mathrm{~mm}(4.6-6.9 \mathrm{~mm})$ (Table 1). In the present cycle, the mean ET at the beginning of hCG treatment (D8) was $5.84 \pm 1.30 \mathrm{~mm}$. After hCG follicular priming (on D14/15), the mean ET significantly increased to $7.61 \pm 2.62 \mathrm{~mm}(\mathrm{p}=0.01$; Table 2$)$. Majority of the patients $(88 \%$ ) experienced more than $10 \%$ improvement after hCG priming (Table 2). About $72 \%$ of the patients had more than $20 \%$ improvement after hCG priming (Table 2). Importantly, 19 out of 25 patients (76\%) achieved an ET more than $7 \mathrm{~mm}$. Thirteen out of 25 patients (52\%) achieved an ET more than $8 \mathrm{~mm}$. Three patients showed no or minimal increase in ET.

Table 1: Impact of hCG follicular priming in patients with thin endometrium $(n=25)$

\begin{tabular}{lllc}
\hline & Before treatment & After hCG & $p$-value \\
\hline $\begin{array}{l}\text { Endometrial } \\
\text { thickness }\end{array}$ & $5.84 \pm 1.30 \mathrm{~mm}$ & $7.61 \pm 2.62 \mathrm{~mm}$ & $<0.01$ \\
Improvement & - & $96 \%$ & $\mathrm{NA}$ \\
$10 \%$ improvement & - & $88 \%$ & $\mathrm{NA}$ \\
$20 \%$ improvement & - & $72 \%$ & $\mathrm{NA}$ \\
Pregnancy rate & 0 & $50 \%$ & $\mathrm{NA}$ \\
\hline
\end{tabular}

NA: Not applicable

Table 2: Comparison of ET in estradiol primed and hCG primed cycles

\begin{tabular}{lllll}
\hline & & $\begin{array}{l}\text { Prev cycle } \\
\text { day 14/15 }\end{array}$ & $\begin{array}{l}\text { Present } \\
\text { cycle D8 }\end{array}$ & $\begin{array}{l}\text { Present cycle } \\
\text { Erev cycle } \\
\end{array}$ \\
& $\begin{array}{l}\text { da D8 (E2 } \\
\text { primed) }\end{array}$ & $\begin{array}{l}\text { ET (E2 } \\
\text { primed) }\end{array}$ & $\begin{array}{l}\text { pre-hCG } \\
\text { ET }\end{array}$ & $\begin{array}{l}\text { D14/15 post- } \\
\text { hCG ET }\end{array}$ \\
\hline Mean & 5.49 & 5.92 & 5.84 & 7.61 \\
Standard & 0.59 & 0.67 & 0.65 & 1.31 \\
deviation & & & & \\
Minimum & 4.1 & 4.6 & 4.4 & 5.00 \\
Maximum & 6.7 & 6.9 & 6.9 & 10.00 \\
\hline
\end{tabular}


Role of Low-dose hCG in Follicular Phase for Thin Endometrium

\begin{tabular}{|c|c|c|c|c|c|c|c|}
\hline Subject & $\begin{array}{l}\text { ET before } h C G \\
(\mathrm{~mm}) \mathrm{D} 8\end{array}$ & Parity & $\begin{array}{l}\text { ET after } h C G \\
(\mathrm{~mm}) \mathrm{D} 14 / 15\end{array}$ & Outcome & Improvement & $10 \%$ & $20 \%$ \\
\hline 1 & 5.6 & POLO & 8.4 & Ongoing 18 weeks+ & Yes & $\mathrm{Y}$ & $\mathrm{Y}$ \\
\hline 2 & 6.0 & POLO & 8.4 & Nonpregnant & Yes & $\mathrm{Y}$ & $\mathrm{Y}$ \\
\hline 3 & 5.3 & POLO & 7.1 & Ongoing & Yes & $\mathrm{Y}$ & $\mathrm{Y}$ \\
\hline 4 & 5.6 & POLO & 8.3 & Nonpregnant & Yes & $\mathrm{Y}$ & $\mathrm{Y}$ \\
\hline 5 & 6.8 & POLO & 8.0 & Nonpregnant & Yes & $\mathrm{Y}$ & $\mathrm{N}$ \\
\hline 6 & 6.0 & POLO & 7.2 & Ongoing & Yes & $\mathrm{Y}$ & Y \\
\hline 7 & 5.1 & POLO & 9.0 & Nonpregnant & Yes & $\mathrm{Y}$ & $\mathrm{Y}$ \\
\hline 8 & 5.5 & POLO & 5.1 & No ET & No & $\mathrm{N}$ & $\mathrm{N}$ \\
\hline 9 & 5.8 & POLO & 7.5 & Pregnant/aborted 20 weeks & Yes & $\mathrm{Y}$ & $\mathrm{Y}$ \\
\hline 10 & 5.9 & POLO & 7.4 & Nonpregnant & Yes & $\mathrm{Y}$ & $\mathrm{Y}$ \\
\hline 11 & 6.9 & POLO & 10.0 & Ongoing & Yes & $\mathrm{Y}$ & $\mathrm{Y}$ \\
\hline 12 & 5.7 & POLO & 8.0 & Ongoing & Yes & $\mathrm{Y}$ & $\mathrm{Y}$ \\
\hline 13 & 5.0 & POLO & 5.8 & No ET & Yes & $\mathrm{Y}$ & $\mathrm{N}$ \\
\hline 14 & 6.0 & POLO & 7.9 & Nonpregnant & Yes & $\mathrm{Y}$ & $\mathrm{Y}$ \\
\hline 15 & 6.2 & POLO & 9.0 & Pregnant/miscarriage & Yes & $\mathrm{Y}$ & $\mathrm{Y}$ \\
\hline 16 & 6.3 & POLO & 8.0 & Nonpregnant & Yes & $\mathrm{Y}$ & $\mathrm{Y}$ \\
\hline 17 & 6.2 & POLO & 7.6 & Nonpregnant & Yes & $\mathrm{Y}$ & $\mathrm{Y}$ \\
\hline 18 & 4.9 & POLO & 8.3 & Nonpregnant & Yes & $Y$ & $Y$ \\
\hline 19 & 5.8 & POLO & 6.0 & No ET & Yes & $\mathrm{N}$ & $\mathrm{N}$ \\
\hline 20 & 5.0 & POLO & 5.4 & No ET & Yes & $\mathrm{N}$ & $\mathrm{N}$ \\
\hline 21 & 6.0 & POLO & 6.9 & Nonpregnant & Yes & $Y$ & $\mathrm{~N}$ \\
\hline 22 & 4.4 & POLO & 5.0 & No ET & Yes & $\mathrm{Y}$ & $\mathrm{N}$ \\
\hline 23 & 6.9 & POLO & 9.0 & Pregnant & Yes & $\mathrm{Y}$ & $Y$ \\
\hline 24 & 6.3 & POLO & 8.1 & Pregnant & Yes & $Y$ & $Y$ \\
\hline 25 & 6.8 & POLO & 9.0 & Pregnant & Yes & $Y$ & $Y$ \\
\hline
\end{tabular}

Twenty-two patients with ET had improvement $>10 \%$, and 18 patients had ET improvement $>20 \%$. Twenty patients had embryo transfer and 10 became pregnant and 8 are ongoing pregnancies. The overall clinical pregnancy rate was $50 \%$. Ongoing pregnancy rate was $40 \%$.

One patient had miscarriage at 20 weeks due to cervical incompetence and the other one had first trimester miscarriage. There was no significant difference in the pregnancy rate between the patients who had ET between 7 and $8 \mathrm{~mm}$ and greater than $8 \mathrm{~mm}$ after hCG administration $(\mathrm{p}=0.41)$.

Specific characteristics of each patient are presented in detail in Table 3.

\section{DISCUSSION}

The current pilot study was an attempt to improve ET in patients with repeatedly thin endometrium (resistant to previous treatments). All patients had been previously treated with either extended, gradually increasing dose of 17-beta estradiol up to $8 \mathrm{mg} /$ day with vaginal sildenafil or human menopausal gonadotropin priming or vitamin E administration but with no success in terms of improvement in endometrium, thereby leading to cancellation of embryo transfer. Our study showed improvement in ET by $20 \%$ in more than $50 \%$ of the patients. The reason for
hCG priming was based on the fact that hCG/luteinizing hormone receptors are present in endometrium. The expression of functional receptors appears to be cycledependent, being present from the proliferative phase, and regulated by changes in the alternative splicing pattern. ${ }^{13}$ Early hCG priming has a positive paracrine effect during luteal phase, and this effect is due to the receptivity of the endometrium, regardless of thickness. Trilaminar morphology of endometrium might be more clinically related to the receptivity capacity of the endometrium than the thickness. ${ }^{14}$ Zhu et a ${ }^{15}$ investigated histological factors related to endometrial receptivity and related them to the pattern of endometrium - trilaminar or homogeneous - in the late follicular phase during natural cycles. They observed that vascular endothelial growth factor (VEGF), integrin alpha and beta levels, as well as fully developed pinopodes were significantly lower in cases with ultrasonographically homogeneous endometrium (not trilaminar) indicating poor receptivity.

Human chorionic gonadotropin, a major embryonic signal, plays a critical role in the initiation and maintenance of pregnancy. To investigate possible direct effects of hCG on endometrial paracrine function in the human female in vivo, Licht et $\mathrm{al}^{16}$ developed an intrauterine microdialysis system that allowed the continuous 
sampling from the uterine cavity over time as well as the application of exogenous hCG and the monitoring of the tissue response to this stimulus. The hCG administration during the secretory phase significantly modulated several endometrial paracrine parameters that correlate with endometrial differentiation (insulin-like growth factor-binding protein 1), angiogenesis VEGF, implantation (leukemia inhibitory factor, macrophage colony-stimulating factor), and tissue remodeling (matrix metalloproteinase 9). ${ }^{16}$ Similarly, Bourdiec et al ${ }^{17}$ investigated whether hCG can modulate endometrial stromal cell (ESC) receptivity to interleukin-1 (IL-1) during the implantation window and have an impact on angiogenesis. Interleukin-1 appears to exert a direct impact on the receptive endometrium and induces major molecular changes that are essential for embryo implantation. The angiogenic activity in vitro was studied using human microvascular endothelial cell line, scratch wound assay, and cell proliferation. They observed that hCG induced a dose-dependent change in ESC receptivity to IL-1 by significantly upregulating the functional signaling receptor IL-1R1 and concomitantly downregulating the decoy inhibitory IL-1R2. Basic research findings like the above mentioned support a paracrine hCG action on endometrium. Hence, in patients with resistant thin endometrium, hCG might really prove beneficial toward enhancing the receptivity of a poorly developed endometrium.

A thorough search through the literature revealed that another group carried out a similar study where 17 infertile patients with successive implantation failures and resistant thin endometrium were recruited. ${ }^{18}$ On day 8 or 9 of the estrogen administration and continuing on $8 \mathrm{mg}$ estrogen per day, subcutaneous injections of 150 IU hCG were initiated daily for 7 days. After a week on hCG priming (day 14 or 15), ET was measured. Mean ET increased from 5.2 to $6 \mathrm{~mm}(\mathrm{p}=0.008) ; 35.3 \%$ of the patients had more than $20 \%$ improvement of their ET after hCG priming. In contrast, $29.4 \%$ patients did not show improvement in ET, whereas $17 \%$ achieved an ET more than $7 \mathrm{~mm}$. Overall, 41\% of them (7/17) finally delivered. Our study showed $72 \%$ patients achieving more than $20 \%$ increment in ET after hCG administration, with overall pregnancy rate of $50 \%$ with ongoing pregnancy rate of $40 \%$. Only three patients $(12 \%)$ showed no or minimal increase in ET after hCG administration.

Another group carried out a similar study in oocyte recipients but having normal endometrium. ${ }^{19}$ They administered much higher dose of hCG (750 IU) every 3 days concomitant to endometrial preparation with estradiol. Sibling oocytes from the same donor were prospectively shared at random among two different recipient groups: Group I where recipients received 750 IU of hCG every 3 days plus estradiol, and group II where recipients received only estradiol. Remarkably, not only ET was significantly lower in group I, but pregnancy rate also was significantly lower in group I as compared with group II (13.6 vs $45.4 \%, \mathrm{p}<0.05$ ). Therefore, the study was discontinued prematurely for ethical reasons when 22 cycles were completed. This scenario indicates a possibility that even hCG beyond a certain dose induces deleterious effect on endometrial receptivity.

One of the strong points in our study design was the fact that we considered improvement in ET only when the thickness increased by $20 \%$. Moreover, we included only patients with resistant thin endometrium (failure of previous medications) of $<7 \mathrm{~mm}$.

Our study is not without limitations. We did not have a control group which received placebo. We have taken previous cycles as a control where ET did not improve in spite of various modifications (estrogens, aspirin, sildenafil). Apart from giving hCG, we used aspirin and/or sildenafil citrate. It can be argued that these could have influenced the results we obtained, but we believe that these could not have essential impact on our results because our patients already had failure with these interventions.

Thin endometrium is a difficult problem in assisted reproduction and it really creates frustration among both doctors and patients. In our pilot study, we achieved almost 50\% pregnancy rate, which indicates the high potential of this protocol.

\section{REFERENCES}

1. Senturk LM, Erel CT. Thin endometrium in assisted reproductive technology. Curr Opin Obstet Gynecol 2008 Jun;20(3): 221-228.

2. Shufaro Y, Simon A, Fatum M. Thin unresponsive endometrium-a possible complication of surgical curettage compromising ART outcome. J Assist Reprod Genet 2008;25:421-425.

3. Fatemi HM, Popovic-Todorovic B, Papanikolaou E, Donoso P, Devroey P. An update of luteal phase support in stimulated IVF cycles. Hum Reprod Update 2007 Nov-Dec;13(6):581-590.

4. Singh P, Hu P, Hoggatt J, Moh A, Pelus LM. Expansion of bone marrow neutrophils following G-CSF administration in mice results in osteolineage cell apoptosis and mobilization of hematopoietic stem and progenitor cells. Leukemia 2012 Nov;26(11):2375-2383

5. Gleicher N, Vidali A, Barad DH. Successful treatment of unresponsive thin endometrium. Fertil Steril 2011 May;95(6): 2123(e13-e17).

6. GleicherN,Kim A,Michaeli T,LeeHJ,Shohat-TalA,LazzaroniE, Barad DH. A pilot cohort study of granulocyte colonystimulating factor in the treatment of unresponsive thin endometrium resistant to standard therapies. Hum Reprod 2013 Jan;28(1):172-177.

7. Kunicki M, Lukaszuk K, Woclawek-Potocka I, Liss J, Kulwikowska P, Szczyptanska J. Evaluation of granulocyte colony-stimulating factor effects on treatment-resistant thin endometrium in women undergoing in vitro fertilization. Biomed Res Int 2014;2014:913235. 
8. Check JH, Cohen R, Choe JK. Failure to improve a thin endometrium in the late proliferative phase with uterine infusion of granulocyte-colony stimulating factor. Clin Exp Obstet Gynecol 2014;41(4):473-475.

9. Barad DH, Yu Y, Kushnir VA, Shohat-Tal A, Lazzaroni E, Lee HJ, Gleicher N. A randomized clinical trial of endometrial perfusion with granulocyte colony-stimulating factor in in vitro fertilization cycles: Impact on endometrial thickness and clinical pregnancy rates. Fertil Steril 2014 Mar;101(3):710-715.

10. Miwa I, Tamura H, Takasaki A, Yamagata Y, Shimamura K, Sugino N. Pathophysiologic features of "thin" endometrium. Fertil Steril 2009 Apr;91(4):998-1004.

11. Acharya S, Yasmin E, Balen AH. The use of a combination of pentoxifylline and tocopherol in women with a thin endometrium undergoing assisted conception therapies-a report of 20 cases. Hum Fertil (Camb) 2009 Dec;12(4):198-203.

12. Takasaki A, Tamura H, Miwa I, Taketani T, Shimamura K, Sugino N. Endometrial growth and uterine blood flow: a pilot study for improving endometrial thickness in the patients with a thin endometrium. Fertil Steril 2010 Apr;93(6):1851-1858.

13. Licht $\mathrm{P}$, von Wolff M, Berkholz A, Wildt L. Evidence for cycle-dependent expression of full-length human chorionic gonadotropin/luteinizing hormone receptor mRNA in human endometrium and decidua. Fertil Steril 2003 Mar;79:(Suppl 1): 718-723.

14. DettiL, YelianFD, Kruger ML, DiamondMP,PuscheckEE. Endometrial thickness dynamics and morphologic characteristics during pituitary downregulation with antagonists in assisted reproductive technology cycles. J Ultrasound Med 2008 Nov; 27(11):1591-1596.

15. Zhu W, Chen M, Liu J, Fu Z, Li X, Qin C, Liu D, Tang X. Receptivity assessment of an ultrasonographic homogeneous endometrium in the late follicular phase of infertile women with natural cycles. Am J Obstet Gynecol 2012 Dec;207(6): 511-517.

16. Licht P, Russu V, Lehmeyer S, Wildt L. Molecular aspects of direct LH/hCG effects on human endometrium-lessons from intrauterine microdialysis in the human female in vivo. Reprod Biol 2001;1:10-19.

17. Bourdiec A, Shao R, Rao CV, Akoum A. Human chorionic gonadotropin triggers angiogenesis via the modulation of endometrial stromal cell responsiveness to interleukin 1: a new possible mechanism underlying embryo implantation. Biol Reprod 2012 Sep;87(3):66.

18. Papanikolaou EG, Kyrou D, Zervakakou G, Paggou E, Humaidan P. Follicular HCG endometrium priming for IVF patients experiencing resisting thin endometrium. A proof of concept study. J Assist Reprod Genet 2013 Oct;30(10): 1341-1345.

19. Prapas N, Tavaniotou A, Panagiotidis Y, Prapa S, Kasapi E, Goudakou M, Papatheodorou A, Prapas Y. Low-dose human chorionic gonadotropin during the proliferative phase may adversely affect endometrial receptivity in oocyte recipients. Gynecol Endocrinol 2009 Jan;25(1):53-59. 